\title{
CT myocardial perfusion imaging: ready for prime time?
}

\author{
Richard A. P. Takx ${ }^{1} \cdot$ Csilla Celeng $^{1} \cdot$ U. Joseph Schoepf ${ }^{2,3}$
}

Received: 30 June 2017 /Revised: 8 August 2017 / Accepted: 5 September 2017 /Published online: 27 September 2017

(C) European Society of Radiology 2017

\begin{abstract}
The detection of functional coronary artery stenosis with coronary CT angiography (CCTA) is suboptimal. Additional CT myocardial perfusion imaging (CT-MPI) may be helpful to identify patients with myocardial ischaemia in whom coronary revascularization therapy would be beneficial. CT-MPI adds incremental diagnostic and prognostic value over obstructive disease on CCTA. It allows for the quantitation of myocardial blood flow and calculation of coronary flow reserve and shows good correlation with ${ }^{15} \mathrm{O}-\mathrm{H}_{2} \mathrm{O}$ positron emission tomography and invasive fractional flow reserve. In addition, patients prefer CCTA/CT-MPI over SPECT, MRI and invasive coronary angiography. CT-MPI is ready for clinical use for detecting myocardial ischaemia caused by obstructive disease. Nevertheless, the clinical utility of CT-MPI to identify ischaemia in patients with non-obstructive/microvascular disease still has to be established.
\end{abstract}

Key Points

- CT-MPI can improve the positive predictive value of CCTA for lesion-specific ischaemia.

- CT-MPI adds incremental prognostic value over CCTA for major adverse cardiac events.

U. Joseph Schoepf

schoepf@musc.edu

1 Department of Radiology, University Medical Center Utrecht, Utrecht, The Netherlands

2 Division of Cardiovascular Imaging, Department of Radiology and Radiological Science, Medical University of South Carolina, Charleston, SC, USA

3 Heart and Vascular Center, Medical University of South Carolina, Ashley River Tower, 25 Courtenay Drive, Charleston, SC 29425-2260, USA
- CT-MPI correlates with ${ }^{15} \mathrm{O}-\mathrm{H}_{2} \mathrm{O}$ PET.

- CT-MPI/CCTA shows high overall patient satisfaction.

Keywords Perfusion · Computed tomography $\cdot$ Coronary artery disease $\cdot$ Ischaemia $\cdot$ Patient satisfaction

\section{Editorial}

Coronary CT angiography (CCTA) is an excellent noninvasive test for ruling out obstructive coronary artery disease (CAD) [1]. Nevertheless, the positive predictive value of CCTA for identifying myocardial ischaemia is suboptimal $[1,2]$. More importantly, CCTA as an a priori anatomical imaging test shares with invasive coronary angiography (ICA) the limitations for accurately gauging the haemodynamic relevance of a lesion; furthermore, severe anatomical narrowing does not necessarily imply the presence of functionally significant stenosis [3].

\section{The role of CT-myocardial perfusion imaging (MPI) in obstructive CAD}

CT myocardial perfusion imaging (CT-MPI) has emerged as a non-invasive imaging method for the detection of myocardial ischaemia [4]. Currently, three different approaches are available to perform CT-MPI: conventional CT-MPI (i.e. snapshot perfusion), dynamic perfusion CT-MPI and dual-energy CTMPI (DECT-MPI) [5]. These different techniques have shown good diagnostic performance [6, 7]. Nevertheless, dynamic and DECT-MPI seem to have a better sensitivity, likely due to the ability to detect more subtle perfusion defects $[8,9]$. Cury et al. [10] showed in a multicentre study that regadenoson CT-MPI was in good agreement with SPECT 
for detecting reversible ischaemia. Results from a sub-analysis of the CORE320 (Combined Non-invasive Coronary Angiography and Myocardial Perfusion Imaging Using 320 Detector Computed Tomography) study [11] demonstrated superior diagnostic accuracy of CT-MPI compared to SPECT for predicting obstructive CAD on ICA. Nevertheless, ICA is a suboptimal test to establish the hemodynamic severity of significant stenosis [12]. In a recent metaanalysis, CT-MPI demonstrated a high diagnostic accuracy for identifying haemodynamically significant myocardial perfusion defects determined by ICA in combination with fractional flow reserve (FFR), with comparable results to magnetic resonance imaging (MRI) [7]. The combination of CT-MPI with CCTA allows for anatomical and functional evaluation of CAD $[13,14]$. The addition of CT-MPI to obstructive lesions on CCTA (e.g. $\geq 50 \%$ stenosis degree) appears to be of incremental value for the diagnosis of myocardial ischaemia as determined by invasive FFR [4]. One multicentre registry evaluated the prognostic value of dynamic CT-MPI [15] for major adverse cardiac events in 144 patients during a followup period up to 18 months; the authors observed that also in prognostication the addition of CT-MPI to obstructive stenosis on CCTA results in an incremental prognostic value, which remained when correcting for clinical risk factors. Furthermore, a clear trend in increase in hazard ratio was observed with an increase in the number of territories with perfusion defects. These results are congruent with studies combing SPECT with CCTA $[16,17]$. Van Rosendael et al. [18] demonstrated not only that those with a normal stress CTMPI had a low occurrence of major cardiovascular events at 12 months, but also that adding CT-MPI to obstructive disease on CCTA results in lower referral rates for ICA and revascularisation.

\section{CT-MPI in non-obstructive CAD and microvascular disease}

CT-MPI allows for the quantitation of myocardial blood flow (MBF) and calculation of coronary flow reserve (CFR) [19, 20]. Abnormal measures can be suggestive for the presence of epicardial and/or microvascular CAD [21]. At least 10-30\% of patients with angina pectoris undergoing ICA have no significant stenosis, and among those 50-65\% are believed to have coronary microvascular dysfunction [22-25]. The recognition of microvascular dysfunction and the potentially underlying myocardial ischaemia is, however, often delayed due to the 'hidden nature' of the disease. Williams et al. evaluated the diagnostic performance of 'snapshot' adenosine stress CTMPI [26]. Part of the study population (22 out of 51) was compared to ${ }^{15} \mathrm{O}-\mathrm{H}_{2} \mathrm{O}$ positron emission tomography (PET). ${ }^{15} \mathrm{O}-\mathrm{H}_{2} \mathrm{O}$ allows for free diffusion (100\% extraction fraction) and is linearly correlated with myocardial uptake [27, 28].
However, its use is mainly limited to research since it requires an on-site cyclotron due to the short half-time of the tracer. In their study, Williams et al. [26] demonstrated that CT myocardial attenuation (measured in Hounsfield units) during hyperaemia correlates with $\mathrm{MBF}$ as measured by ${ }^{15} \mathrm{O}-\mathrm{H}_{2} \mathrm{O}$ PET. The observed difference in ${ }^{15} \mathrm{O}-\mathrm{H}_{2} \mathrm{O}$ uptake between no $\mathrm{CAD}$ and non-obstructive $\mathrm{CAD}$ under hyperaemic conditions shows potential to detect microvascular disease. In addition, CT-MPI/CCTA demonstrated a good positive predictive value of $90 \%$ on a patient level compared to ICA/FFR. With regard to gender, women are more prone to have nonobstructive CAD compared to men; the WISE (Women's Ischemia Syndrome Evaluation) study showed that $81 \%$ of women referred for ICA have no or non-obstructive CAD [29]. At a 10-year follow-up cardiovascular death or myocardial infarction occurred in $6.7 \%$ of women with minimal CAD (i.e. $\leq 20 \%$ diameter reduction) and in $12.8 \%$ of women with non-obstructive CAD (i.e. $>20 \%$, but $<50 \%$ narrowing) [30]. Due to the relatively high occurrence of events in patients with non-obstructive CAD, the incorporation of CT-MPI to clinical decision-making can be of great importance for better identification of myocardial ischaemia.

\section{Patient satisfaction and radiation dose}

A recent study by Feger et al. [31] observed high patient satisfaction for CT (including CT-MPI) and found that patients prefer it over SPECT, MRI and ICA. In detail, half of the patients preferred the combined CT-MPI/CCTA approach, with only $2 \%$ of patients giving preference to stress MRI. Furthermore, the use of (semi)-automated quantification of CT-MPI data provides substantially reduced analysis times, making it feasible to integrate quantitative CT-MPI into clinical workflow [32]. A concern with CT-MPI is the associated increase in radiation exposure of patients. In a review, Danad et al. [9] calculated an average radiation exposure of $5.9 \mathrm{mSv}$ (range 1.9-15.7) for snapshot CT-MPI and $9.2 \mathrm{mSv}$ (range 3.8-12.8) for dynamic CT-MPI. Nevertheless, when using low-kV protocols dynamic CT-MPI is feasible at $4-6 \mathrm{mSv}$ $[33,34]$. Adenosine is usually well tolerated by patients, though the administration is of concern for patients with advanced heart block or asthma, and patients should avoid caffeine intake $24 \mathrm{~h}$ before testing [35]. The recent introduction of third generation DSCT and dual layer CT systems is expected to further increase the role of CT-MPI with highly accurate iodine quantification [36]. Furthermore, data from the SPECIFIC (Dynamic Stress Perfusion CT for Detection of Inducible Myocardial Ischemia) trial, which aims to determine the diagnostic accuracy of CT-MPI compared with invasive FFR in patients with suspected or known CAD (ClinicalTrials. gov Identifier: NCT02810795) is expected to give more insight into the role of dynamic CT-MPI. 


\section{Conclusion}

CT-MPI is a proven method for detecting myocardial ischaemia caused by obstructive CAD, is associated with high patient satisfaction, and has been shown to be ready for clinical use in this setting. Recent research shows that perfusion imaging has incremental prognostic value over stenosis degree on CCTA. In addition, it has the potential to identify ischaemia caused by non-obstructive CAD and microvascular disease. Nevertheless, the future clinical utility of CT-MPI to identify the extent of ischaemia in patients with non-obstructive/microvascular CAD still has to be established.

Funding The authors state that this work has not received any funding.

\section{Compliance with ethical standards}

Guarantor The scientific guarantor of this publication is U. Joseph Schoepf.

Conflict of interest The authors of this manuscript declare relationships with the following companies: Richard A.P. Takx has nothing to disclose.

Csilla Celeng is supported by the European Association of Cardiovascular Imaging (EACVI) Research Grant.

U. Joseph Schoepf is a consultant for and receives research support from Astellas, Bayer, GE, Guerbet, Medrad and Siemens.

Statistics and biometry No complex statistical methods were necessary for this paper.

Ethical approval Institutional Review Board approval was not required because the manuscript is an Editorial.

Methodology Editorial

\section{References}

1. Menke J, Kowalski J (2016) Diagnostic accuracy and utility of coronary $\mathrm{CT}$ angiography with consideration of unevaluable results: A systematic review and multivariate Bayesian randomeffects meta-analysis with intention to diagnose. Eur Radiol 26: $451-458$

2. Dharampal AS, Papadopoulou SL, Rossi A et al (2013) Diagnostic performance of computed tomography coronary angiography to detect and exclude left main and/or three-vessel coronary artery disease. Eur Radiol 23:2934-2943

3. Ahmadi A, Stone GW, Leipsic J et al (2016) Association of Coronary Stenosis and Plaque Morphology With Fractional Flow Reserve and Outcomes. JAMA Cardiol 1:350-357

4. Osawa K, Miyoshi T, Miki T et al (2016) Diagnostic Performance of First-Pass Myocardial Perfusion Imaging without Stress with Computed Tomography (CT) Compared with Coronary CT Angiography Alone, with Fractional Flow Reserve as the Reference Standard. PLoS One 11:e0149170

5. Ruzsics B, Lee H, Zwerner PL, Gebregziabher M, Costello P, Schoepf UJ (2008) Dual-energy CT of the heart for diagnosing coronary artery stenosis and myocardial ischemia-initial experience. Eur Radiol 18:2414-2424
6. Delgado Sanchez-Gracian C, Oca Pernas R, Trinidad Lopez C et al (2016) Quantitative myocardial perfusion with stress dual-energy $\mathrm{CT}$ : iodine concentration differences between normal and ischemic or necrotic myocardium. Initial experience. Eur Radiol 26:31993207

7. Takx RA, Blomberg BA, El Aidi H et al (2015) Diagnostic accuracy of stress myocardial perfusion imaging compared to invasive coronary angiography with fractional flow reserve meta-analysis. Circ Cardiovasc Imaging 8:e002666

8. Bamberg F, Hinkel R, Schwarz F et al (2012) Accuracy of dynamic computed tomography adenosine stress myocardial perfusion imaging in estimating myocardial blood flow at various degrees of coronary artery stenosis using a porcine animal model. Invest Radiol 47:71-77

9. Danad I, Szymonifka J, Schulman-Marcus J, Min JK (2016) Static and dynamic assessment of myocardial perfusion by computed tomography. Eur Heart J Cardiovasc Imaging 17:836-844

10. Cury RC, Kitt TM, Feaheny K et al (2015) A randomized, multicenter, multivendor study of myocardial perfusion imaging with regadenoson CT perfusion vs single photon emission CT. J Cardiovasc Comput Tomogr 9:e101-e102

11. George RT, Mehra VC, Chen MY et al (2014) Myocardial CT perfusion imaging and SPECT for the diagnosis of coronary artery disease: a head-to-head comparison from the CORE320 multicenter diagnostic performance study. Radiology 272:407-416

12. Uren NG, Melin JA, De Bruyne B, Wijns W, Baudhuin T, Camici PG (1994) Relation between myocardial blood flow and the severity of coronary-artery stenosis. N Engl J Med 330:1782-1788

13. Ko BS, Cameron JD, Meredith IT et al (2012) Computed tomography stress myocardial perfusion imaging in patients considered for revascularization: a comparison with fractional flow reserve. Eur Heart J 33:67-77

14. Ko BS, Cameron JD, Leung M et al (2012) Combined CT coronary angiography and stress myocardial perfusion imaging for hemodynamically significant stenoses in patients with suspected coronary artery disease: a comparison with fractional flow reserve. JACC Cardiovasc Imaging 5:1097-1111

15. Meinel FG, Pugliese F, Schoepf UJ et al (2017) Prognostic Value of Stress Dynamic Myocardial Perfusion CT in a Multicenter Population With Known or Suspected Coronary Artery Disease. AJR Am J Roentgenol 208:761-769

16. Pazhenkottil AP, Nkoulou RN, Ghadri JR et al (2011) Prognostic value of cardiac hybrid imaging integrating single-photon emission computed tomography with coronary computed tomography angiography. Eur Heart J 32:1465-1471

17. van Werkhoven JM, Schuijf JD, Gaemperli O et al (2009) Prognostic value of multislice computed tomography and gated single-photon emission computed tomography in patients with suspected coronary artery disease. J Am Coll Cardiol 53:623-632

18. van Rosendael AR, Dimitriu-Leen AC, de Graaf MA et al (2017) Impact of computed tomography myocardial perfusion following computed tomography coronary angiography on downstream referral for invasive coronary angiography, revascularization and, outcome at 12 months. Eur Heart J Cardiovasc Imaging. https://doi. org/10.1093/ehjci/jex055

19. Christian TF, Frankish ML, Sisemoore JH et al (2010) Myocardial perfusion imaging with first-pass computed tomographic imaging: Measurement of coronary flow reserve in an animal model of regional hyperemia. J Nucl Cardiol 17:625-630

20. Coenen A, Lubbers MM, Kurata A et al (2017) Diagnostic value of transmural perfusion ratio derived from dynamic CT-based myocardial perfusion imaging for the detection of haemodynamically relevant coronary artery stenosis. Eur Radiol 27:2309-2316

21. van de Hoef TP, Siebes M, Spaan JA, Piek JJ (2015) Fundamentals in clinical coronary physiology: why coronary flow is more important than coronary pressure. Eur Heart J 36:3312-3319a 
22. Reis SE, Holubkov R, Lee JS et al (1999) Coronary flow velocity response to adenosine characterizes coronary microvascular function in women with chest pain and no obstructive coronary disease. Results from the pilot phase of the Women's Ischemia Syndrome Evaluation (WISE) study. J Am Coll Cardiol 33:1469-1475

23. Graf S, Khorsand A, Gwechenberger M et al (2007) Typical chest pain and normal coronary angiogram: cardiac risk factor analysis versus PET for detection of microvascular disease. J Nucl Med 48: 175-181

24. Murthy VL, Naya M, Foster CR et al (2011) Improved cardiac risk assessment with noninvasive measures of coronary flow reserve. Circulation 124:2215-2224

25. Marinescu MA, Loffler AI, Ouellette M, Smith L, Kramer CM, Bourque JM (2015) Coronary microvascular dysfunction, microvascular angina, and treatment strategies. JACC Cardiovasc Imaging 8:210-220

26. Williams MC, Mirsadraee S, Dweck MR et al (2016) Computed tomography myocardial perfusion vs $15 \mathrm{O}$-water positron emission tomography and fractional flow reserve. Eur Radiol. https://doi.org/ 10.1007/s00330-016-4404-5

27. Klein R, Beanlands RS, deKemp RA (2010) Quantification of myocardial blood flow and flow reserve: Technical aspects. J Nucl Cardiol 17:555-570

28. Kikuchi Y, Oyama-Manabe N, Naya M et al (2014) Quantification of myocardial blood flow using dynamic 320-row multi-detector CT as compared with (1)(5)O-H(2)O PET. Eur Radiol 24:15471556

29. Pepine CJ, Anderson RD, Sharaf BL et al (2010) Coronary microvascular reactivity to adenosine predicts adverse outcome in women evaluated for suspected ischemia results from the National Heart,
Lung and Blood Institute WISE (Women's Ischemia Syndrome Evaluation) study. J Am Coll Cardiol 55:2825-2832

30. Sharaf B, Wood T, Shaw L et al (2013) Adverse outcomes among women presenting with signs and symptoms of ischemia and no obstructive coronary artery disease: findings from the National Heart, Lung, and Blood Institute-sponsored Women's Ischemia Syndrome Evaluation (WISE) angiographic core laboratory. Am Heart J 166:134-141

31. Feger S, Rief M, Zimmermann E et al (2015) Patient satisfaction with coronary CT angiography, myocardial CT perfusion, myocardial perfusion MRI, SPECT myocardial perfusion imaging and conventional coronary angiography. Eur Radiol 25:2115-2124

32. Ebersberger U, Marcus RP, Schoepf UJ et al (2014) Dynamic CT myocardial perfusion imaging: performance of $3 \mathrm{D}$ semi-automated evaluation software. Eur Radiol 24:191-199

33. Kim SM, Kim YN, Choe YH (2013) Adenosine-stress dynamic myocardial perfusion imaging using 128-slice dual-source CT: optimization of the CT protocol to reduce the radiation dose. Int $\mathrm{J}$ Cardiovasc Imaging 29:875-884

34. Fujita M, Kitagawa K, Ito T et al (2014) Dose reduction in dynamic CT stress myocardial perfusion imaging: comparison of 80-kV/370$\mathrm{mAs}$ and 100-kV/300-mAs protocols. Eur Radiol 24:748-755

35. Botvinick EH (2009) Current methods of pharmacologic stress testing and the potential advantages of new agents. J Nucl Med Technol 37:14-25

36. Pelgrim GJ, van Hamersvelt RW, Willemink MJ et al (2017) Accuracy of iodine quantification using dual energy CT in latest generation dual source and dual layer CT. Eur Radiol. https://doi. org/10.1007/s00330-017-4752-9 